\title{
Unveiling the Nature of Charge Carrier Interactions by Electroabsorption Spectroscopy: An Illustration with Lead-Halide Perovskites
}

\author{
Marine E. F. Bouduban $\S^{\star}$, Andrés Burgos-Caminal, Joël Teuscher, and Jacques-E. Moser
}

§SCS-DSM Award for best poster presentation in Physical Chemistry

\begin{abstract}
Unravelling the nature of the interactions between photogenerated charge carriers in solar energy conversion devices is key to enhance performance. In this perspective, we discuss electroabsorption spectroscopy (EAS), as the spectral bandshape of the electroabsorption (EA) signal directly depends on the strength of the charge carrier interactions. For instance, the electroabsorption response in molecular or confined excitonic systems can be modelled perturbatively yielding the Stark effect. In contrast, most solids exhibit weaker interactions, and a perturbative approach cannot be taken in general. For solids with negligible charge carrier interactions, one resorts to the Franz-Keldysh theory of a continuum in a field, that, in the low-field limit, simplifies to the low-field FKA effect. Alternatively, when the continuum approximation breaks down, the problem of a Wannier exciton in a field has to be solved, and numerical methods emerged as the best solution. We illustrate our discussion with two examples involving lead-halide perovskites, a new, high-stake solar cell material. In the first example, we discuss the lineshape of the electroabsorption response for thin-films of lead-iodide perovskite, that sustains the photogeneration of free carriers. In the second example, we address a confined excitonic case with lead-bromide perovskite nanoparticles, and demonstrate the presence of so-called charge-transfer excitons.
\end{abstract}

Keywords: Charge carrier interactions · Electroabsorption · Lead-halide perovskites · Photovoltaic devices . Spectroscopy

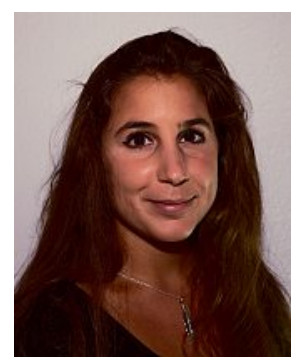

in EPFL, where she studies the ultrafast dynamics of charge carriers in perovskite solar cells aside fundamental excited-state dynamics investigations.

\section{Introduction}

1.1 Electroabsorption Spectroscopy Applied to Photovoltaic Systems

Charge pairs of opposite sign are created upon absorption of light in semiconducting materials. Conduction band electrons and positive holes in the valence band constitute mobile charge carriers. Within the field of photovoltaics, photogenerated transport mechanisms, the possible involvement of trap sites, and the dynamics of interfacial carriers lie at the focus of attention, as their fate directly determines the performance of solar energy conversion devices. In such a context, two questions are of particular interest: the first one relates to the nature of the interaction between light-induced charges. These can stand as free independent carriers, correlated electron-hole pairs with different degrees of interaction, and Coulomb-bound states, such as excitons. The second question addresses the motion of photocarriers
${ }^{\star}$ Correspondence: M. E. F. Boudubar

Institute of Chemical Sciences \& Engineering, and

Lausanne Centre for Ultrafast Science

Eccole polytechnique fédérale de Lausanne

$\mathrm{CH}-1015$ Lausanne

E-mail: marine.bouduban@epfl.ch within and between the materials constituting photovoltaic devices. This includes various charge transfer at donor-acceptor heterojunctions.

Various methods exist that can probe the nature of the interaction and the transport of photogenerated charge carriers. Among them, a particularly interesting combination involves Transient Absorption Spectroscopy (TAS), and Electroabsorption Spectroscopy (EAS), along with its time-resolved extension (Time-Resolved Electroabsorption Spectroscopy, TREAS). The well-established TAS technique is particularly useful to monitor the kinetics of interfacial charge transfer processes. Alternatively, EAS allows to determine the strength of the interaction between photogenerated carriers by lineshape analysis, while the newly-developed TREAS technique affords a handle for real time monitoring of the separation of photocarriers in materials submitted to an electric field. EAS and TREAS have been successfully applied to scrutinize the dynamics of photocarriers in small molecule- and conjugated polymer-based organic photovoltaic (OPV) systems..$^{[1-3]}$ They are currently believed to constitute very powerful tools for the study of another 
class of compounds of interest: lead-halide perovskites. ${ }^{[4]}$

The term perovskite originally designated calcium titanate $\mathrm{CaTiO}_{3}$ and is now generalized to all compounds that exhibit an $\mathrm{ABX}_{3}$ crystal structure in the form of corner-sharing $\mathrm{BX}_{6}$ octahedra with interstitial A cations for charge neutralization. In the context of lead-halide perovskites, $\mathrm{B} \equiv \mathrm{Pb}^{2+}, \mathrm{X} \equiv \mathrm{Cl}^{-}, \mathrm{Br}^{-}$, or $\mathrm{I}^{-}$and $\mathrm{A}$ is an (in)organic cation, such as $\mathrm{CH}_{3} \mathrm{NH}_{3}{ }^{+}, \mathrm{CH}_{2}(\mathrm{NH})_{2}{ }^{+}$, $\mathrm{Cs}^{+}$, or $\mathrm{Rb}^{+}$. These particular perovskites constitute high-stake materials, due to their remarkable optoelectronic properties: their application to solar cells (perovskite solar cells, PSCs), LED devices and lasing have led to outstanding performances. ${ }^{[5-16]}$ Such a wide range of applications implies a wide range of shapes and structure, and, indeed, although mostly exploited in the form of semiconductor thin-films, lead-halide perovskites can be found as nanocrystals or colloidal nanoparticles, retaining their 3D geometry, as well as quantum-confined, 2D or quasi-2D layers.

In spite of important research efforts, some of the key properties of perovskite materials are still unclear. In particular, the interactions between photogenerated charge carriers and their involvement in transport and recombination dynamics are yet to be completely understood. For instance, transient absorbance signals recorded with perovskite thin-films exhibit excitonic signatures, in accordance with what one would expect from an ionic semiconductor $(\mathrm{Pb}-\mathrm{X}$ bonds $(\mathrm{X}=\mathrm{Cl}, \mathrm{Br}$, I) have only a weak covalent character). As excitons are neutral quasiparticles formed through the Coulomb interaction between an electron and a positive hole, this electrostatic interaction is indeed expected to be stronger in ionic media that are characterized by a low dielectric constant. Conversely, the electronic polarizability of covalent solids generally results in a high electric susceptibility and an efficient screening of the Coulomb interactions between carriers. Now, numerous studies have yielded values of the exciton binding energy in perovskites and, although results show a great variability, they all converge to surprisingly low values of $2-60 \mathrm{meV}$ for $\mathrm{CH}_{3} \mathrm{NH}_{3} \mathrm{PbI}_{3}$ and up to $150 \mathrm{meV}$ for $\mathrm{CH}_{3} \mathrm{NH}_{3} \mathrm{PbBr}_{3},{ }^{[17-23]}$ indicating that mostly free carriers are generated at room temperature. In addition, it has been shown that the precise character of the photogenerated carriers, as well as the ratio of free carriers to bound electron-hole pairs are highly dependent upon the nanostructuring of the material.

In the following, we briefly review the context of the electroabsorption response, that is the optical behavior of a physicochemical system submitted to an electric field. We then explain how EAS can help unravel the character of photogenerated carriers, focusing on lead-halide perovskite systems that are relevant for photovoltaic applications.

\subsection{Electroabsorption Spectroscopy: Background and Embodiment from Molecular to Solid-state Physics}

Electroabsorption spectroscopy belongs to the family of the 'differential' techniques, where the signal is defined as the difference between the optical properties of a sample in presence and in absence of a perturbation. In the present case (Eqn. (1)), the perturbation is an externally applied or photoinduced (local) electric field, and the signal monitored at a wavelength $\lambda$ is defined as an absorbance change $\Delta A(\lambda)$ :

$$
\Delta A(\lambda)=A_{\text {field }}(\lambda)-A_{\text {no field }}(\lambda)
$$

It emerges that the spectral shape and amplitude of the electroabsorption signal is directly related to the response of the sample upon application of a field. When an electric field is applied on a quantum system, it induces a reorganization of its electronic density and hence a modification of the nuclear configuration, ultimately modifying the electronic structure. ${ }^{[24]}$ As a consequence, the electroabsorption response could be obtained theoretically by solving the delicate problem of a quantum hydrogenic system in a field, for which no analytical solution exists. The functional form of the solution is thus highly dependent on the type of approximation employed, and hence this response will differ tremendously when considering a solid or a molecular system. We briefly survey these two different cases in the following sections.

\subsubsection{EAS of Molecules or Confined Excitonic States}

In the context of molecules or confined excitonic states, the Coulomb interaction within the hydrogenic model is large, and the applied electric field can be considered negligible. As a consequence, one can resort to a perturbative solution, that is, the response of the system of interest in the field can be modelled in the framework of Stark theory. The absorbance change is then given by a perturbative expansion (Eqn. (2)):[25]

$$
\begin{aligned}
& \Delta A(\lambda)=-\frac{\partial A(\lambda)}{\partial \lambda} \cdot \boldsymbol{m}_{0 \mathrm{k}} \cdot \boldsymbol{E}-\frac{1}{2} \cdot \frac{\partial A(\lambda)}{\partial \lambda} \\
& \boldsymbol{p}_{0 \mathrm{k}} \cdot \boldsymbol{E}^{2}+\frac{1}{2} \cdot \frac{\partial^{2} A(\lambda)}{\partial \lambda^{2}} \cdot\left(\boldsymbol{m}_{0 \mathrm{k}} \cdot \boldsymbol{E}\right)^{2},
\end{aligned}
$$

where $\boldsymbol{E}$ denotes the externally applied vectorial field, while $\boldsymbol{m}_{0 \mathrm{k}}$ and $\boldsymbol{p}_{0 \mathrm{k}}$ respectively correspond to the permanent dipole moment and polarizability changes upon the optical transition of interest $(0 \rightarrow \mathrm{k})$.

As the first term is linear in the field, it cancels out for isotropic samples. As a result the response of a molecular or confined excitonic system subjected to an electric field is quadratic in the field intensity $|\boldsymbol{E}|$ (quadratic Stark effect). In addition, it can be decomposed into a linear combination of first and second derivatives of the linear absorption spectrum, whose relative weight provides direct insight into the type of photogenerated carriers. Light absorption by charge-transfer (CT) excitons, occurring from the Coulomb interactions of an electron and a hole separated by an interface, for instance, is characterized by a change in the permanent dipole moment. The contribution of these CT states to the electroabsorption response can, thus, be directly inferred from the weight of the second-derivative term.

\subsubsection{Electroabsorption of Solids}

In the case of solids, the assumption of a small perturbative applied field cannot be made generally, as the electrostatic interactions are overall significantly smaller than for the confined case, rendering the use of a perturbative solution highly inappropriate. In addition, a general solution for solid-state systems is a tricky problem, as the level of classical correlation of the electron-hole pairs, i.e. the amplitude of the Coulomb interaction, varies significantly from one system to another. This variation indeed depends on the intrinsic properties of the solid-state system of interest, such as the nature of its chemical bonds and, hence, its electric susceptibility (related to the dielectric constant), which directly determines to which extent electron-hole pairs are correlated. For example, ionic solids exhibit a weaker wavefunction overlap and are characterized by a wider bandgap and a lower susceptibility than their covalent analogs. [26]

From the above, a broad spectrum of interactions emerges, starting with the simplest model to describe a solid-state system: a continuum of single-particle states where electrons and holes are by definition uncorrelated. Then, if from this point, we adiabatically switch on the correlation, we progressively move towards the other extreme of this spectrum where lies the exciton, the bound-pair state solution to the Wannier equation.

The problem of a continuum in an electric field has been tackled by Franz and Keldysh in the 1950s. ${ }^{[27-29]}$ They showed that the resulting eigenfunctions were not plane waves but Airy functions, accounting for the observed electroabsorption signals, scaling as $|\boldsymbol{E}|^{-2 / 3}$ and involving both a redshift (photon-assisted tunneling between bands) and oscillations above the band 
edge. Interestingly, experiments originally carried out on GaAs ${ }^{[30-35]}$ have revealed a different field-dependence in the limit of low field intensities. In this case, the electroabsorption signal scales as $|\boldsymbol{E}|^{2}$, similarly to the Stark effect and is modeled by the so-called low-field Franz-Keldysh-Aspnes (FKA) effect. Importantly, while the Stark effect is determined by a combination of first and second derivatives of the absorption spectrum, the low-field FKA effect appears to depend upon its third derivative.

The continuum model breaks down when the electron-hole interaction becomes significant, ultimately leading to the formation of so-called Wannier-Mott excitons. This calls for other theoretical treatments, namely the excitonic electroabsorption theories. As stated before, no analytical solution exists for the Schrödinger equation of a Wannier exciton in an electric field, and excitonic electroabsorption theories rely on two different approaches: they either resort to approximations (for example to model the external potential) to go back to an analytically solvable problem ${ }^{[37,38]}$ or focus on the development of numerical resolution routines. ${ }^{[26,36,39-42]}$

\section{Unravelling the Properties of Photocarriers using EAS: An Experimental Illustration with Lead-halide Perovskites}

The various types of electroabsorption response described in the previous section exhibit well-defined field-dependences. Their spectral bandshapes also differ: For instance, the Stark effect is characterized by an EA spectrum, which displays a mixture of first- and second-derivatives of the absorption spectrum, while the lowfield FKA effect corresponds to the third derivative. As a consequence, a spectral bandshape analysis of the electroabsorption signal constitutes a probe of the degree of correlation between photocarriers, and hence, of the type of charged particle or quasiparticle involved.

As a first example, we consider the case of lead-iodide perovskite thin-films. A typical electroabsorption spectrum for such a system is shown by Fig. 1. This EA response has been previously assigned to a quadratic Stark effect, thus arising from the presence of confined excitons. ${ }^{[43]}$

However, such an assignment is questionable, notably due to the detailed work by Ziffer et al., in which EAS of thinfilm of methylammonium lead-iodide perovskite was carried out. ${ }^{[36]}$ In this context, it was shown that the obtained EA response at room temperature was better described by the low-field FKA model. This observation is compatible with a low exciton binding energy and indicates that a majority of photocarriers are actually free. This finding is further supported by the calculation of carriers reduced mass and Elliott's function-based exciton binding energy and band gap yielding values in close agreement with other experimental and theoretical results.

As a second example, we now address the case of confined excitons in $\mathrm{CH}_{3} \mathrm{NH}_{3} \mathrm{PbBr}_{3}$ perovskite nanoparticles suspended in chlorobenzene. The preparation of these colloids in the presence of octylammonium surfactant cations yields a broad distribution of nanostructures composed of bulk-like tri-dimensional perovskite spherical nanoparticles and

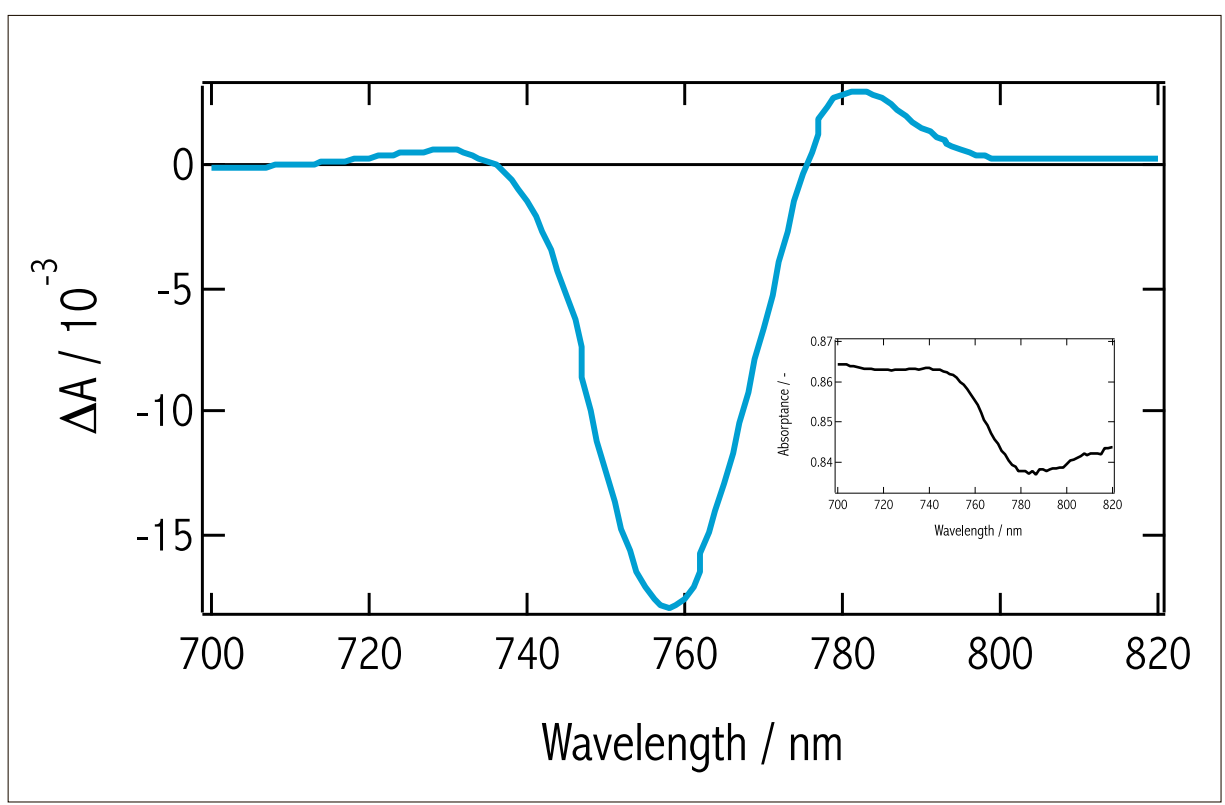

Fig. 1. Electroabsorption spectrum of a $300 \mathrm{~nm}$-thick film of $\mathrm{CH}_{3} \mathrm{NH}_{3} \mathrm{Pbl}_{3}$ perovskite submitted to an externally applied electric field of intensity $|\boldsymbol{E}|=0.70 \pm 0.05 \times 10^{5} \mathrm{~V} \mathrm{~cm}^{-1}$. Inset: Absorptance spectrum of a $300 \mathrm{~nm}$-thick film of $\mathrm{CH}_{3} \mathrm{NH}_{3} \mathrm{Pbl}_{3}$.

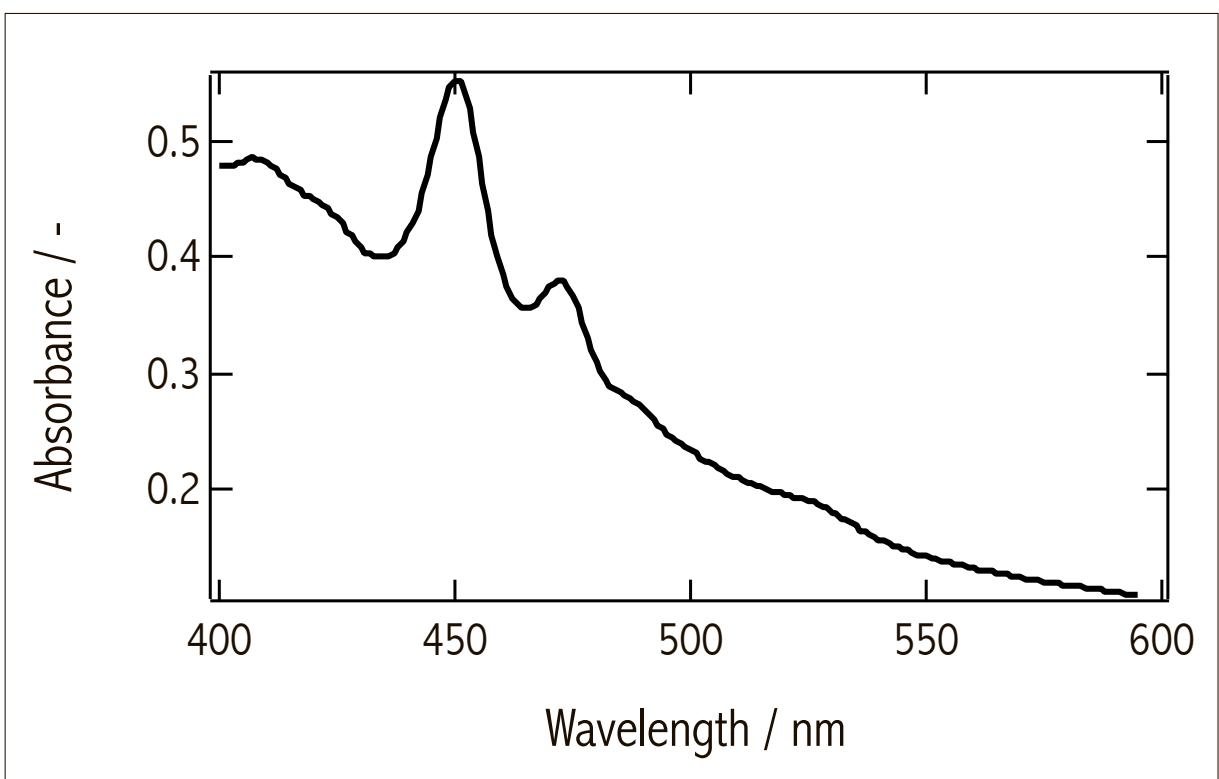

Fig. 2. Absorption spectrum of a colloidal suspension of $\mathrm{CH}_{3} \mathrm{NH}_{3} \mathrm{PbBr}_{3}$ perovskite nanoparticles in chlorobenzene. nanoplatelets of various thicknesses. ${ }^{[43-47]}$ The latter are denominated 'quasi-2D' (q2D), as they exhibit a quasi-two-dimensional structure comprising several (3-5) layers of perovskite unit cells, stabilized by a capping layer of octylammonium cations. Each of these nanostructures exhibits a unique optical signature, observable from their absorbance spectrum as shown in Fig. 2.

Ultrafast transient absorption experiments were performed with the colloidal suspensions. The obtained time-resolved spectra revealed strong oscillations on the blue side of the spectrum on top of the expected bleaching feature at the wavelength 
$\lambda=525 \mathrm{~nm}$. To shed light on the former feature, which looks very much like the first or second derivative of the absorption spectrum of q-2D nanoplatelets over the $400-480 \mathrm{~nm}$ wavelength region, a transient spectrum recorded at a time-delay of 2 ps is shown in Fig. 3A, along with a simulated differential electroabsorption spectrum (Fig. 3B). The correspondence between the measured and simulated differential spectra allows to assign a significant part of the transient optical features observed below $500 \mathrm{~nm}$ to a photoinduced electroabsorption phenomenon. Note that here, rather than being due to an externally-applied electric field, the EA results from the pho- togeneration of carriers yielding localized electric fields within each electron-hole pair.

As discussed in Section 1, the differential electroabsorption response for confined excitonic or molecular systems can be modelled by Eqn. (2) (Stark theory). The signal shown in Fig. 3A is best fitted by a linear combination of first and second derivative terms, corresponding to Eqn. (3).

$$
\Delta A(\lambda)=\frac{\partial A(\lambda)}{\partial \lambda}+10 \cdot \frac{\partial^{2} A(\lambda)}{\partial \lambda^{2}}
$$
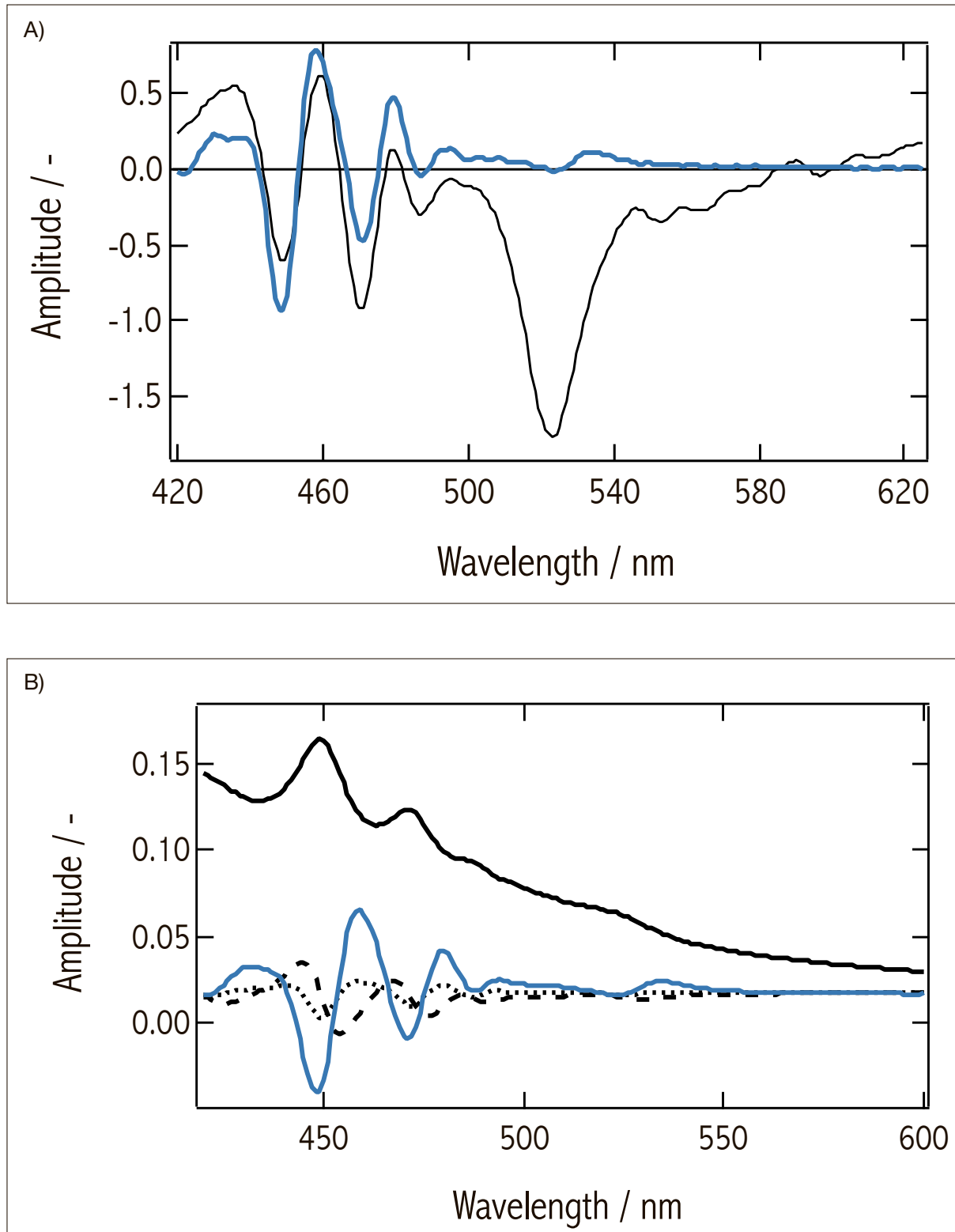

Fig. 3. A) Modeled electroabsorption signal of a suspension of $\mathrm{CH}_{3} \mathrm{NH}_{3} \mathrm{PbBr}_{3}$ nanoparticles in chlorobenzene. Displayed is a superposition of the transient absorption spectrum recorded at a time-delay of 2 ps (black line) and a simulated electroabsorption signal (blue line) corresponding to Eqn. (3). B) Linear absorption spectrum of $\mathrm{CH}_{3} \mathrm{NH}_{3} \mathrm{PbBr}_{3}$ nanoparticles (black solid line) together with its first (dashed line) and second (dotted line) derivatives. The best fitted model (blue line) is obtained as a linear combination of first and second derivatives with Eqn. (3).
The electroabsorption signal, then, appears to be clearly dominated by the second derivative of the linear absorption spectrum, whose amplitude is given by the change in permanent dipole moment $\boldsymbol{m}_{\mathrm{ok}}$ occurring during the optical transition (see Eqn. (2)).

This last finding is of great importance, as it allows to determine which type of photocarriers originated the EA signal. Contrary to free carriers and Frenkel and Wannier-Mott excitons, interfacial chargetransfer (CT) excitons exhibit a permanent dipole moment. ${ }^{[24]}$ The observed EA signal, therefore, evidences the presence of CT states at the interface between nanostructured domains constituted by individual q-2D platelets and 3D particles. This is not only interesting from a fundamental perspective but it has also an important implication for the photovoltaic application, as the understanding of inter-domain interactions within multigrain perovskite films, as well as carrier trapping and recombination within CT excitons is key to the optimization of PSCs photovoltaic performances.

\section{Conclusion}

The two examples discussed here, leadiodide perovskite thin-films and perovskite nanoparticles, illustrate how a simple spectral bandshape analysis of the electroabsorption response allows useful insights to be gained into the photogenerated carriers and the strength of their interactions. In the first case, the assignment of the roomtemperature EA signal to a low-field FKA effect provides evidence towards the dominance of free carriers shortly after photoexcitation. In the second case, within the framework of Stark theory, the observation of a second derivative-dominated electroabsorption signal in a transient absorption measurement on perovskite nanoparticles endorses the presence of interfacial charge-transfer excitons. In both cases, the obtained outcomes are of great interest, as they allow one step forward to be taken in the understanding of the underlying photophysics in perovskite systems, paving the way towards more efficient photovoltaic devices.

\section{Acknowledgements}

Financial support by the National Center of Competence in Research 'Molecular Ultrafast Science and Technology' (NCCR MUST), an instrument of the Swiss National Science Foundation, is gratefully acknowledged.

Received: February 7, 2017 
[1] A. Devižis, D. Hertel, K. Meerholz, V. Gulbinas, J. E. Moser, Org. Electronics 2014, 15, 3729.

[2] A. Devižis, J. De Jonghe-Risse, R. Hany, F. Nüesch, S. Jenatsch, V. Gulbinas, J. E. Moser, J. Am. Chem. Soc. 2015, 137, 8192.

[3] M. Causa', J. De Jonghe-Risse, M. Scarongella, J. C. Brauer, E. Buchaca-Domingo, J.-E. Moser, N. Stingelin, N. Banerji, Nat. Commun. 2016, 7 , 12556.

[4] A. A. Paraecattil, J. Risse-De Jonghe, V. Pranculis, J. Teuscher, J.-E. Moser, J. Phys. Chem. C 2016, 120, 19595.

[5] J. Xing, F. Yan, Y. Zhao, S. Chen, H. Yu, Q. Zhang, R. Zeng, H. V. Demir, X. Sun, A. Huan, Q. Xiong, ACS Nano 2016, 10, 6623.

[6] N. Wang, L. Cheng, R. Ge, S. Zhang, Y. Miao, W. Zou, Nat. Photon. 2016, 10, 699 .

[7] S. D. Stranks, H. J. Snaith, Nat. Nanotech $\mathbf{2 0 1 5}, 10,391$.

[8] F. Zhang, H. Zhong, C. Chen, X.-G. Wu, X. Hu, H. Huang, J. Han, B. Zou, Y. Dong, ACS Nano $\mathbf{2 0 1 5}, 9,4533$

[9] M. Saliba, T. Matsui, J.-Y. Seo, K. Domanski, J.-P. Correa-Baena, M. K. Nazeeruddin, S. M. Zakeeruddin, W. Tress, A. Abate, A. Hagfeldt, M. Grätzel, Energy Environ. Sci. 2016, 9, 1989.

[10] D. Bi, W. Tress, M. I. Dar, P. Gao, J. Luo, C. Renevier, K. Schenk, A. Abate, F. Giordano, J. P. Correa Baena, J. D. Decoppet, S. M. Zakeeruddin, M. K. Nazeeruddin, M. Grätzel, A. Hagfeldt, Sci. $A d v$. 2016, 2, e1501170.

[11] J. P. C. Baena, L. Steier, W. Tress, M. Saliba, S. Neutzner, T. Matsui, F. Giordano, T. J. Jacobsson, A. R. S. Kandada, S. M Zakeeruddin, A. Petrozza, A. Abate, M. K. Nazeeruddin, M. Grätzel, A. Hagfeldt, Energy Environ. Sci. 2015, 8, 2928.

[12] M. Grätzel, Nat. Mater. 2014, 13, 838.

[13] H. J. Snaith, J. Phys. Chem. Lett. 2013, 4, 3623

[14] W. K. Chong, K. Thirumal, D. Giovanni, T. W. Goh, X. Liu, N. Mathews, S. Mhaisalkar, T. C. Sum, Phys. Chem. Chem. Phys. 2016, 18, 14701.
[15] S. Bai, Z. Yuan, F. Gao, J. Mater. Chem. C 2016, 4, 3898.

[16] S. W. Eaton, M. Lai, N. A. Gibson, A. B. Wong, L. Dou, J. Ma, L.-W. Wang, S. R. Leone, P. Yang, Proc. Natl Acad. Sci. USA 2016, 113 , 1993.

[17] K. Tanaka, T. Takahashi, T. Ban, T. Kondo, K. Uchida, N. Miura, Solid State Commun. 2003, 127,619 .

[18] J. H. Heo, S. H. Im, J. H. Noh, T. N. Mandal, C.-S. Lim, J. A. Chang, Y. H. Lee, H.-J. Kim, A. Sarkar, M. K. Nazeeruddin, M. Grätzel, S. Il Seok, Nat. Photon. 2013, 7, 1.

[19] T. M. Brenner, D. A. Egger, A. M. Rappe, L. Kronik, G. Hodes, D. Cahen, J. Phys. Chem. Lett. 2015, 6, 4754.

[20] M. Hirasawa, T. Ishihara, T. Goto, K. Uchida, N. Miura, Physica B 1994, 201, 427.

[21] A. M. Soufiani, F. Huang, P. Reece, R. Sheng, A. Ho-Baillie, M. A. Green, Appl. Phys. Lett. 2015, 107, 231902.

[22] S. Sun, T. Salim, N. Mathews, M. Duchamp, C. Boothroyd, G. Xing, T. C. Sum, Y. M. Lam, Energy Environ. Sci. 2014, 7, 399.

[23] A. Miyata, A. Mitioglu, P. Plochocka, O. Portugall, J. T.-W. Wang, S. D. Stranks, H. J. Snaith, R. J. Nicholas, Nat. Phys. 2015, 11, 582.

[24] H. Nakatsuji, T. Hayakawa, T. Yonezawa, J. Am. Chem. Soc. 1981, 103, 7426.

[25] G. Lanzani, 'The Photophysics behind Photovoltaics and Photonics', Wiley-VCH, Weinheim, 2012.

[26] D. F. Blossey, Phys. Rev. B 1970, 2, 3976.

[27] W. Franz, Z. Naturforsch. A 1958, 13, 484.

[28] L. V. Keldysh, J. Exptl. Theoret. Phys. (USSR) 1957, 33, 994; translation: Soviet Physics JETP 1958, 6,763 .

[29] L. V. Keldysh, J. Exptl. Theoret. Phys. (USSR) 1964, 47, 1945; translation: Soviet Physics JETP 1965, 20, 1307.

[30] D. E. Aspnes, J. E. Rowe, Phys. Rev. B 1971, 5 , 4022.
[31] D. E. Aspnes, Surf. Sci. 1973, 37, 418.

[32] D. E. Aspnes, A. Frova, Solid State Commun. 1969, 7,155

[33] D. E. Aspnes, Solid State Commun. 1970, 8 , 267.

[34] A. Frova, P. Handler, F. A. Germano, D. E. Aspnes, Phys. Rev. B 1966, 145, 575.

[35] M. Cardona, in 'Festkörperprobleme 10', vol. 10, Springer, Heidelberg, 1970, p. 125.

[36] M. E. Ziffer, J. C. Mohammed, D. S. Ginger, ACS Photon. 2016, 3, 1060.

[37] C. B. Duke, M. E. Alferieff, Phys. Rev. 1966 145, 583.

[38] C. M. Penchina, J. K. Pribram, J. Sak, Phys. Rev. 1969, 188, 1240.

[39] H. I. Ralph, J. Phys. C: Solid State Phys. 1968, 1,378 .

[40] J. D. Dow, D. Redfield, Phys. Rev. B 1970, 1, 3358.

[41] D. F. Blossey, Phys. Rev. B 2016, 1382.

[42] M. T. Trinh, X. Wu, D. Niesner, X. Y. Zhu, $J$. Mater. Chem. A 2015, 3, 9285.

[43] K. Zheng, Q. Zhu, M. Abdellah, M. E. Messing, W. Zhang, A. Generalov, Y. Niu, L. Ribaud, S. E. Canton, T. Pullerits, J. Phys. Chem. Lett. 2015, 6, 2969.

[44] Y. Hassan, Y. Song, R. D. Pensack, A. I Abdelrahman, Y. Kobayashi, M. A. Winnik, G. D. Scholes, Adv. Mater. 2015, 28, 566.

[45] B. Luo, Y.-C. Pu, Y. Yang, S. A. Lindley, G. Abdelmageed, H. Ashry, Y. Li, X. Li, J. Z Zhang, J. Phys. Chem. C 2015, 119, 26672.

[46] O. Vybornyi, S. Yakunin, M. V. Kovalenko, Nanoscale 2016, 8, 6578

[47] P. Tyagi, S. M. Arveson, W. A. Tisdale, J. Phys. Chem. Lett. 2015, 6, 1911. 